\title{
Imaging Psychogenic Movement Disorders
}

\author{
Arpan R. Mehta • James B. Rowe • Anette E. Schrag
}

Published online: 22 September 2013

(C) The Author(s) 2013. This article is published with open access at Springerlink.com

\begin{abstract}
The neurobiological basis of psychogenic movement disorders (PMDs) has been elusive, and they remain difficult to treat. In the last few years, functional neuroimaging studies have provided insight into their pathophysiology and neural correlates. Here, we review the various methodological approaches that have been used in both clinical and research practice to address neural correlates of functional disorders. We then review the dominant hypotheses generated from the literature on psychogenic paralysis. Overall, these studies emphasize abnormalities in the prefrontal and anterior cingulate cortices. Recently, functional neuroimaging has been used to specifically examine PMDs. These studies have addressed a
\end{abstract}

This article is part of the Topical Collection on Neuroimaging

\section{A. R. Mehta \\ Division of Clinical Neurology, Nuffield Department of Clinical \\ Neurosciences, University of Oxford, Oxford, UK \\ e-mail: arpan.mehta@ndcn.ox.ac.uk}

\section{J. B. Rowe}

Department of Clinical Neurosciences, University of Cambridge, Cambridge, UK

e-mail: james.rowe@mrc-cbu.cam.ac.uk

\section{J. B. Rowe}

Medical Research Council Cognition and Brain Sciences Unit, Cambridge, UK

\section{J. B. Rowe}

Behavioural and Clinical Neuroscience Institute, Cambridge, UK

\author{
A. E. Schrag \\ Institute of Neurology, Royal Free Campus, \\ University College London, London, UK
}

\section{A. E. Schrag $(\bowtie)$}

UCL Institute of Neurology, University College London, Royal Free Campus, Clinical Neurosciences, Rowland Hill Street,

London NW3 2PF, UK

e-mail: a.schrag@ucl.ac.uk major point of controversy: whether higher frontal brain areas are directly responsible for inhibiting motor areas or whether they reflect modulation by attentional and/or emotional processes. In addition to elucidating the mechanism and cause, recent work has also explored the lack of agency that characterizes PMDs. We describe the results and implications of the results of these imaging studies and discuss possible interpretations.

Keywords Psychogenic movement disorder · Functional disorder · Motor conversion - Somatoform disorder .

Dissociative disorder $\cdot$ Medically unexplained $\cdot$ Neurological symptom $\cdot$ Functional imaging $\cdot$ Functional magnetic resonance imaging $\cdot$ Positron emission tomography $\cdot$ Single photon emission computed tomography $\cdot$ Motor $\cdot$ Cerebellum . Striatum $\cdot$ Basal ganglia $\cdot$ Prefrontal cortex $\cdot$ Attention · Emotion $\cdot$ Agency

\section{Introduction}

Psychogenic movement disorders (PMDs) are movement disorders thought to result from a psychological or psychiatric, as opposed to a primarily neurological, disturbance. The term 'functional movement disorders' has also been used, to reflect the frequent absence of a psychological trigger and the current understanding of these disorders (see [1] for a recent review). However, here we will use the term 'psychogenic', in keeping with the nomenclature used in most imaging studies of these disorders [2]. Although the diagnosis of PMDs technically includes the voluntary production of the illness characteristics (i.e. malingering or factitious disorders, in which symptoms are attributable to external or internal gains, respectively), such cases are rare [3-5] and most are thought to be due to involuntary processes. PMDs have been described as a "crisis for neurology" [6]: they are very common [7-9], challenging 
to diagnose $[10,11]$, difficult to treat effectively $[12-15]$ and associated with a poor prognosis [16-19]. The limited understanding of their psychopathophysiology underlies the difficulties in their management $[20,21]$. Insights from neuroimaging studies are therefore important for tackling the major problem of PMDs.

In recent years, neuroimaging has begun to elucidate the mechanisms and neural correlates of PMDs. This includes the neuroanatomical basis for PMDs, and the identification of dysfunctional processing and network interactions in brain areas related to sensorimotor control, attention and emotion.

\section{Neuroimaging Methods: Advantages and Limitations}

Several different neuroimaging methods have been used to study psychogenic neurological disorders, each with their specific properties. In clinical practice, structural imaging primarily has a role as a 'negative diagnostic marker' by detecting the absence of gross neurological disease [22]. There is a relative paucity of research using structural techniques to examine the specific anatomy of psychogenic neurological disorders [23]. Clinical use of functional imaging modalities (positron emission tomography, PET, and single photon emission computed tomography, SPECT) includes the differentiation of Parkinson's disease from psychogenic parkinsonism [24, 25]. However, although a negative dopamine transporter SPECT scan in the setting of a parkinsonian syndrome can be very useful to confirm impairment of the dopaminergic pathways, it does not completely exclude a diagnosis of organic parkinsonism, e.g. for patients with 'scans without evidence of dopaminergic deficit' [26-28], or for those with drug-induced parkinsonism [1]. It is also recognized that psychogenic and organic parkinsonism can coexist [29], and patients with psychogenic parkinsonism may therefore require longitudinal follow-up [3].

In contrast to clinical imaging, functional magnetic resonance imaging (fMRI) has been widely used for research studies, in addition to PET and SPECT. These methods have been used to characterize the neural correlates and establish neurobiological models for psychogenic neurological disorders. Functional magnetic resonance imaging (fMRI) has the advantages of lack of radiation, higher spatial and temporal resolution than PET or SPECT and greater potential for modelling neural systems, owing to the scale and richness of fMRI datasets. However, PET and SPECT offer analysis of perfusion, arguably a more direct marker of local neurometabolic rates than values inferred from the blood oxygen level dependent contrast of fMRI. It is also easier to study 'natural' movements by allowing participants to perform motor tasks outside the scanner or immediately prior to scanning, during the period when the radiotracer is injected. Different analytical approaches have been adopted, such as statistical parametric mapping and scaled subprofile mapping, reflecting the need for measures of both localization and integration of neural systems. For the former, brain areas that are significantly different in activation between conditions are identified via analysis of covariance on a voxel-by-voxel basis, whereas in the latter, a variant of principal component analysis is performed that identifies covariant neural network activity. In addition, functional connectivity analyses have allowed inferences to be made about the coupling of task-related states with changes in neural interactions. These methods include psychophysiological interactions in general linear models (PPI) [30], dynamic causal modelling and Granger causality modelling [31, 32]. The latter has been adopted to evaluate the directionality of effects, determining whether activity in one brain area causes changes in a candidate target region. Functional imaging by magnetoencephalography has yet to be used to study PMDs, and the excellent spatial and temporal resolution of magnetoencephalography mean that it is a potentially powerful tool to study the complex temporal dynamics and disordered network integration in PMDs.

Although detecting changes in neural activation and networks can be informative about neural mechanisms, it cannot, in isolation, explain the psychological mechanisms of PMDs. It might be tempting to conclude that differences in brain activity cause the psychogenic disorder, but there are at least two other possible interpretations: that they are the effect of the illness, or that they reflect compensatory changes in the neural networks. Nonetheless, neuroimaging can generate testable hypotheses, encouraging further study using other research tools, such as behavioural and electrophysiological methods [33-40].

A challenge for studies of psychogenic neurological disorders, and particularly for studies of PMDs, has been the identification of homogeneous groups of patients to allow group analysis. There are marked differences in affected body areas, the type and combination of neurological presentation, the psychological trigger and the characteristics of the movement disorder. Functional neuroimaging has been used to examine individuals or groups of patients with various sensory and motor psychogenic neurological disorders (Table 1) $[41-54,55 \bullet, 56 \bullet, 57 \bullet, 58,59,60 \bullet$. The focus has been, until recently, predominantly on negative motor symptoms, such as psychogenic paralysis. Although one cannot easily extrapolate the findings to PMDs, this provided the benefit of removing some of the methodological and analytical challenges of studying patients with differing hyperkinetic states. Moreover, there is considerable overlap between psychogenic paralysis and PMDs in many patients [61•]. We will therefore first consider the conclusions from studies of patients with psychogenic paralysis.

\section{Insights from Psychogenic Paralysis}

The comparison of studies on psychogenic paralysis is limited by small sample sizes and methodological heterogeneity: 


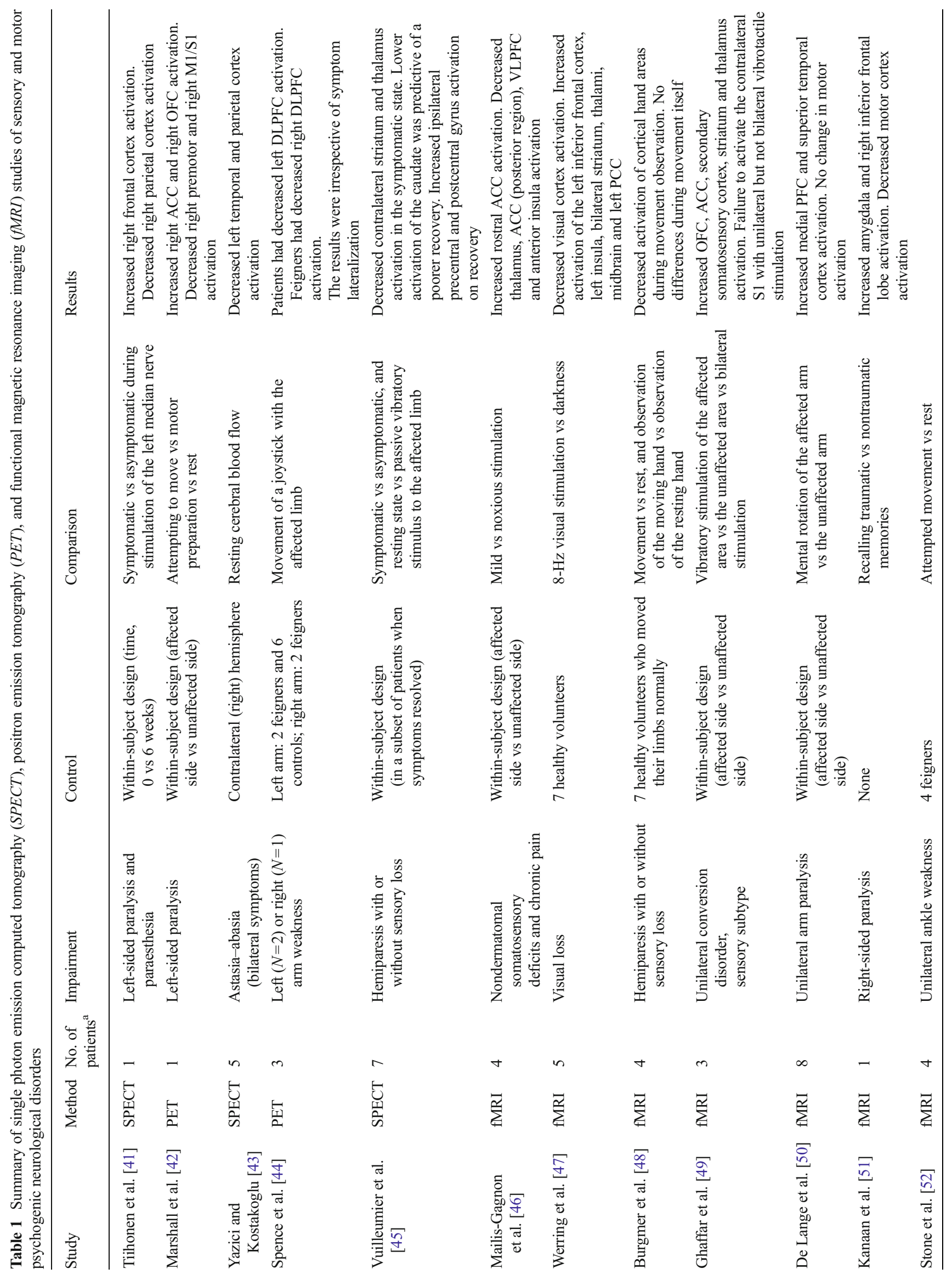




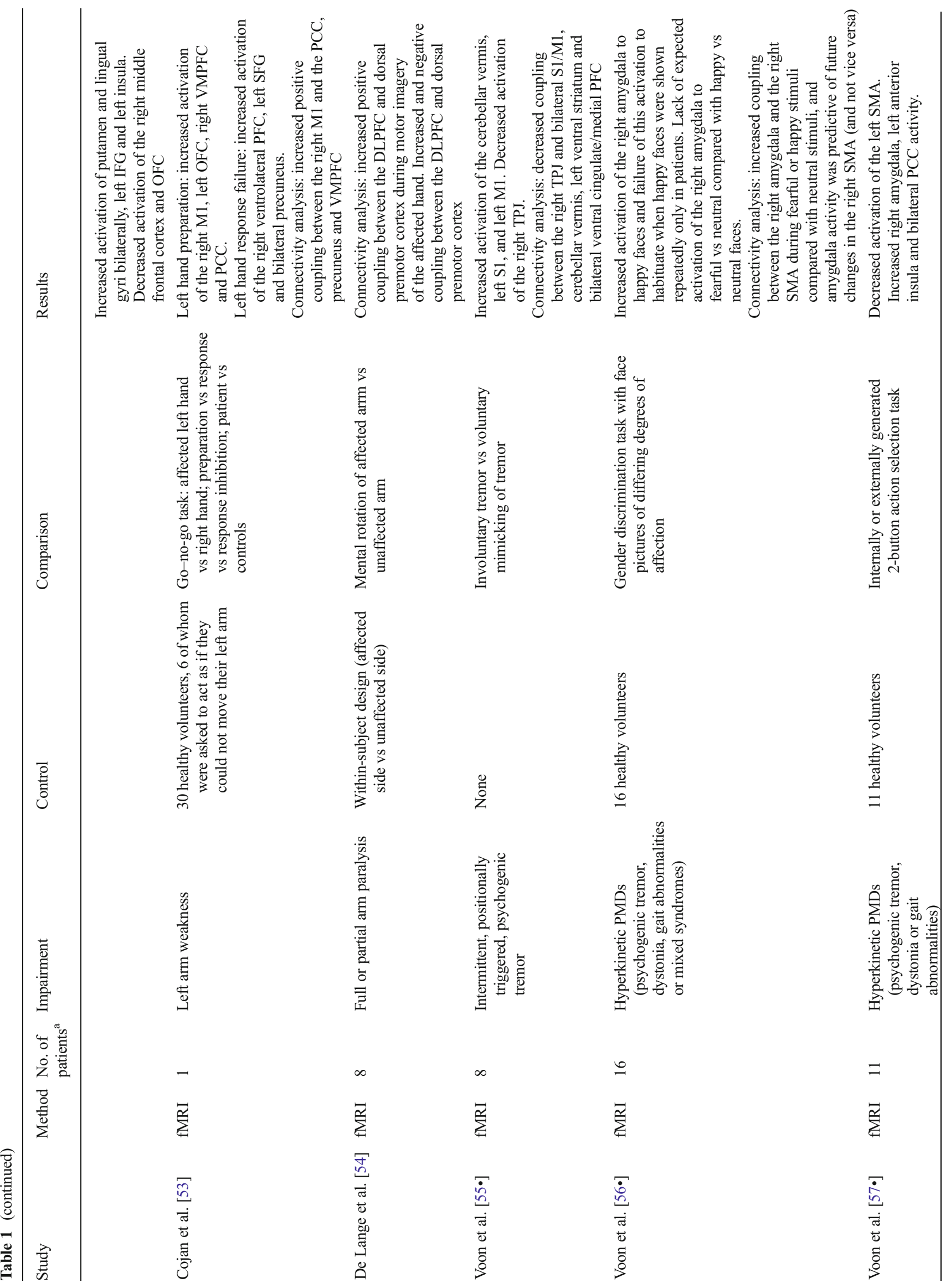




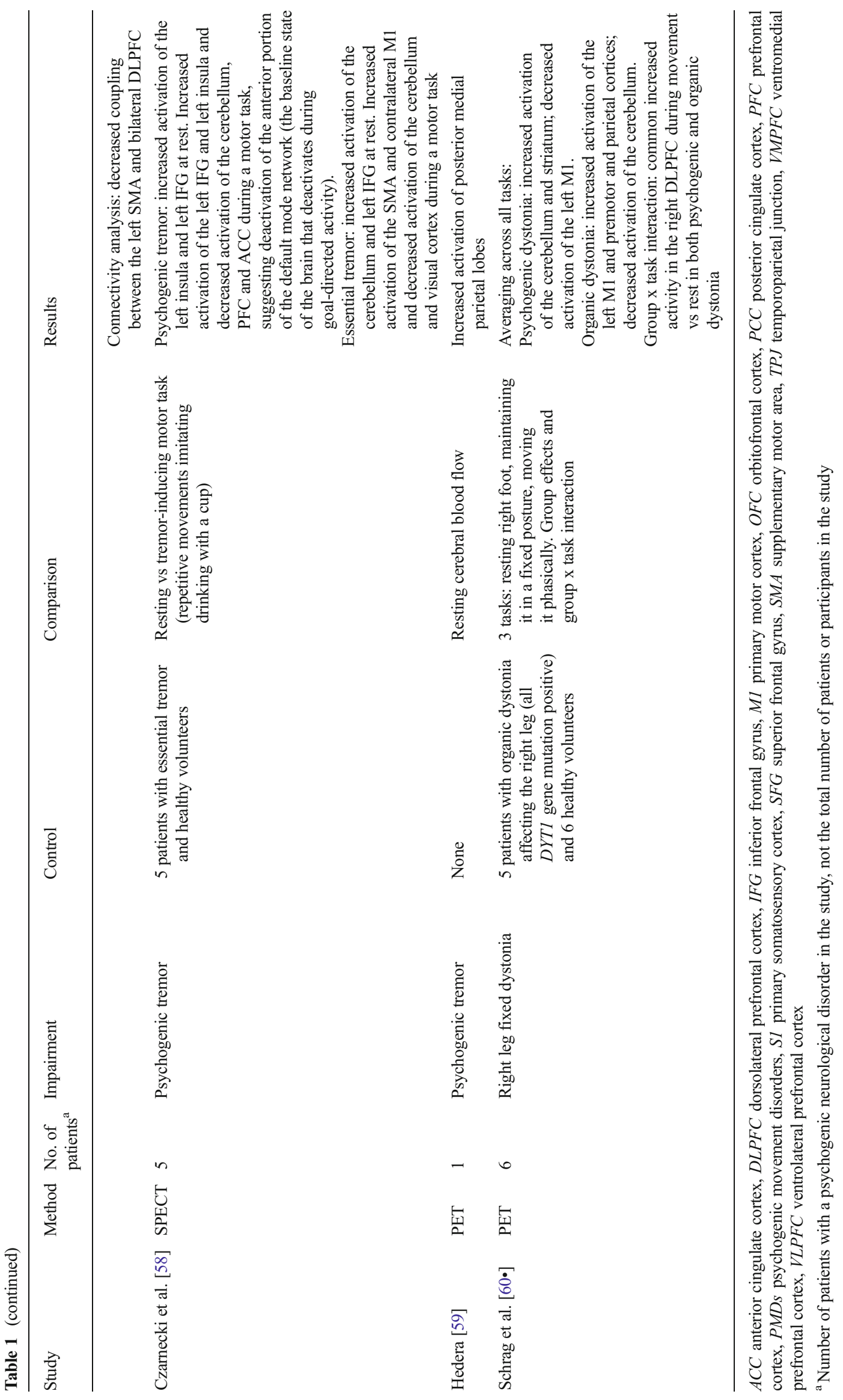


different experimental paradigms, different durations of the deficit (acute versus chronic) and different participant enrolment criteria with respect to comorbidities, such as depression and chronic pain. Most have used motor tasks that are close to the functional deficit [62], such that participants have been asked to try to move the affected limb, imagine moving the paralysed limb or feign immobility of the unaffected limb [42, $44,50,63,64]$. This can lead to potential confounds related to differing degrees of motor performance and sensory feedback. Studies have also recruited volunteers who may be psychologically or neurobiologically distinct from patients who do not agree with their diagnosis of a psychogenic disorder or who choose not to engage in research [3].

Nevertheless, there is a body of neuroimaging evidence on the pathophysiology of these disorders. Overall, these accounts emphasize abnormalities outside the core motor network, including the prefrontal cortex and anterior cingulate cortex. Several key hypotheses have emerged:

1. There is impaired generation of motor intention $[44,48$, 65].

2. There is impairment of motor conceptualization [66].

3. There is disruption of motor execution $[45,53,67]$.

4. Patients have impaired self-monitoring $[50,53]$.

5. There are deficits in limbic processing [42, 45, 63, 64].

6. There is dysregulation of 'top-down' control from higherorder frontal regions [41, 51, 54].

The major point of controversy is whether higher frontal brain areas are directly responsible for inhibiting motor areas or whether they reflect modulation by attentional or emotional processes [2]. Vuilleumier [68] pointed out that previous studies could not parse out the role of limbic activity in the inhibitory process because of confounding factors, such as the stress that a patient may experience secondary to trying to move a subjectively paralysed leg. Vuilleumier et al. [45] addressed this issue by investigating patients with psychogenic hemisensory loss, measuring blood flow via SPECT during tuning fork stimulation of the affected and unaffected limbs in both the active phase and the recovered phase of their illness. They showed that hypoactivation of the thalamus and striatum contralateral to the affected limb normalized with the patients' recovery from their illness. They concluded that pathogenic striatothalamocortical circuits play a primary role in the pathogenesis of these disorders. More recently, Cojan et al. [53] used fMRI to examine a single patient with psychogenic paralysis compared with 24 controls and six feigners of paralysis during a go-no-go task performed with both affected (left) and unaffected hands. The patient had normal preparatory activation in the right motor cortex, indicating preserved motor intentions, but there were concomitant increases in activation in ventromedial prefrontal cortical regions. During failure to execute movement in go trials with the affected hand, there was activation in the precuneus and ventrolateral frontal gyrus, but no activation of right frontal areas normally subserving inhibition. There was, however, increased activation in these areas during no-go trials for the unaffected hand. In contrast, the healthy controls who were asked to feign hemiparalysis showed similar activation in both go and nogo trials for the affected hand, suggesting that distinct inhibitory mechanisms are implicated in simulation and conversion paralysis. Edwards et al. [61•] point out that the specific region of the ventromedial prefrontal cortex that was active in this study dovetails precisely with the region proposed by Burgess et al. [69] to allow sustained, self-maintained, attentional mediation. In contrast to Cojan et al., de Lange et al. [54] performed PPI analysis examining the coupling between the different nodes of the prefrontal cortex and sensorimotor areas and did not demonstrate connectivity between the ventromedial prefrontal cortex and the motor network during their motor imagery task (imaging or visualizing an action without performing it). Instead, they found increased negative coupling between the dorsolateral prefrontal cortex and sensorimotor areas, and increased positive coupling between the dorsolateral prefrontal cortex and dorsolateral premotor cortex. The strength of the coupling was greater when patients imagined movements of their affected, compared with their unaffected, hand. The two studies provide evidence for frontal areas modulating motor activity in a more complex manner than pure motor inhibition.

\section{Neuroimaging of PMDs}

Despite the potential clinical similarities between psychogenic paralysis and PMDs, the latter are associated with positive phenomena rather than solely a lack of movement. Thus, their pathophysiology is unlikely to be purely related to inhibition of movement. There are fewer studies specifically of PMDs $[54,55 \bullet, 56 \bullet, 57 \bullet, 58,59,60 \bullet]$, but they have tended to recruit larger cohorts, and have increased awareness of the clinical, psychological and neural heterogeneity amongst patients.

Several key questions concerning the pathophysiology of PMDs have been posed. How are the symptoms generated (mechanism); why are the symptoms generated (cause); and why is the symptom experienced as being involuntary (touching on the concept of agency) [34, 70-72]? Neuroimaging has contributed to the emerging understanding of all three functions in PMDs: mechanism, cause, and agency.

Motor control could be impaired at several stages: planning, execution or inhibition. It could also be modulated by abnormal top-down processes such as attentional mechanisms [35] or affective mechanisms, as suggested by the clinical observation that distraction often normalizes symptoms. To address this, Voon et al. [56•] performed an elegant study using a task with different affective stimuli, presenting them to patients with PMDs and healthy controls. The principal innovation was that the task was unrelated to the motor deficit, 
thereby circumventing issues related to impaired motor task performance and focusing on neurocognitive systems for emotional processing and arousal, including the amygdala. The choice of task was particularly pertinent, given the influences of emotional events on the initiation or maintenance of psychogenic neurological disorders [73]. Patients showed similar levels of activation in the right amygdala for fearful faces as healthy controls, but increased activation for happy faces. Furthermore, this activation failed to habituate in patients when happy faces were shown repeatedly. This finding is consistent with reports that show that such patients have greater startle reflexes to arousing stimuli [74], and that patients with psychogenic neurological disorders display a failure of habituation of galvanic skin responses to acoustic stimuli $[75,76]$. In addition, using two methods of connectivity (PPI and Granger causality modelling), they showed that patients viewing emotionally charged stimuli compared with neutral stimuli had abnormal connectivity between the amygdala and the supplementary motor area, and that the likely direction of influence was from the amygdala to motor regions. This was the first study to show that patients with PMDs have greater neural responses to arousal, along with increased interactions between the brain regions associated with arousal and motor control. It therefore provides a framework for explaining both the cause and the maintenance of PMD symptoms.

In a further study, Voon et al. [57•] examined whether brain regions associated with motor initiation are affected during voluntary action selection in patients with PMDs compared with healthy volunteers. Eleven patients with PMDs and 11 healthy volunteers performed either an internally or an externally generated two-button action selection task during fMRI. When performing both internally and externally generated movements, the patients with PMDs had abnormally low activity of the supplementary motor area and increased activity of the right amygdala and the left anterior insula and bilateral posterior cingulate cortices. Thus, there was lower activity in regions associated with motor preparation and higher activity in limbic structures during motor preparation, suggesting a possible mechanism of aberrant emotional processing impacting on normal motor planning. Moreover, during internally versus externally generated action selection, the left supplementary motor area had lower functional connectivity with bilateral dorsolateral prefrontal cortices in patients compared with controls, providing evidence for impaired topdown regulation of internally generated action selection. Together, the two studies suggest that emotionally arousing events might provoke an aberrant action selection response from the supplementary motor area, which is both hypoactive and functionally disconnected from prefrontal top-down regulation.

The perception of involuntariness is clear despite evidence that the movements are generated by the same motor network as for voluntary actions [77]: the abnormal movements do not have the normal sense of intention or ownership that is associated with our usual voluntary movement [33, 34]. For example, psychogenic movements, such as functional myoclonus, are preceded by a Bereitschaftspotential ('readiness potential'), whose origin includes regions such as the premotor cortex, supplementary motor area and primary motor cortex [78]. Furthermore, psychogenic tremor can be entrained, i.e. the frequency of the tremor takes on the same frequency of voluntary rhythmic movements in another body part, suggesting that neural oscillators involved in voluntary movement and PMDs interact and converge on common pathways [77]. A recent fMRI study addressed the involuntary nature of the symptoms of PMDs. Voon et al. [55•] found lower activity in the right temporoparietal junction in patients with psychogenic tremor, compared with activations evoked by asking the patients to simulate their tremor voluntarily. This area is thought to be a comparator of actual and predicted sensory feedback. Curiously, this finding goes against studies that have demonstrated increased activity in this area in conditions when movements feel involuntary in both normal healthy people [79, 80] and patients with schizophrenia [81]. However, they also demonstrated lower functional connectivity between this region and sensorimotor regions and limbic regions, a finding that potentially explains the anomaly [61•]. Voon et al. [55•] proposed that their findings might represent a failure in PMDs to match the actual and predicted sensory feedback, giving rise to a feeling in the patients that their movements are involuntary.

There have been few neuroimaging studies that compare the abnormal activation in PMDs with their organic counterpart [58, 60•]. Such comparisons are essential for inferences about causality, necessity and sufficiency: there may be similarly abnormal brain activation patterns in other psychogenic and organic movement disorders, including in the motor and limbic neural systems.

To address this, our group compared the functional neuroimaging correlates of psychogenic and organic dystonia [60•]. Similarities and differences of regional cerebral blood flow were compared in a carefully selected group of patients with

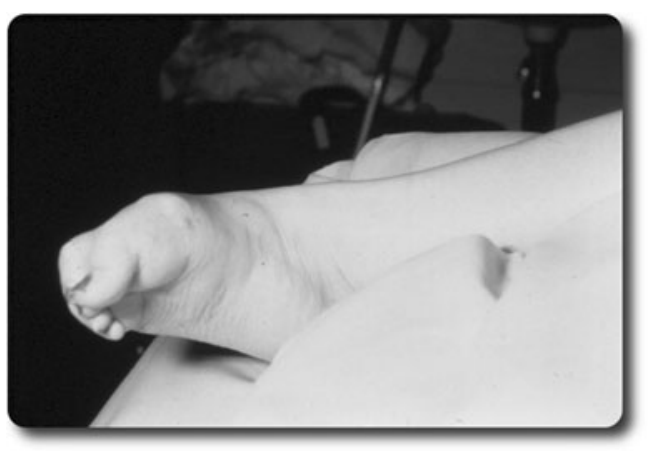

Fig. 1 The syndrome of fixed dystonia of the lower limb. (Reproduced with permission of Oxford University Press from: Schrag et al. [12]) 

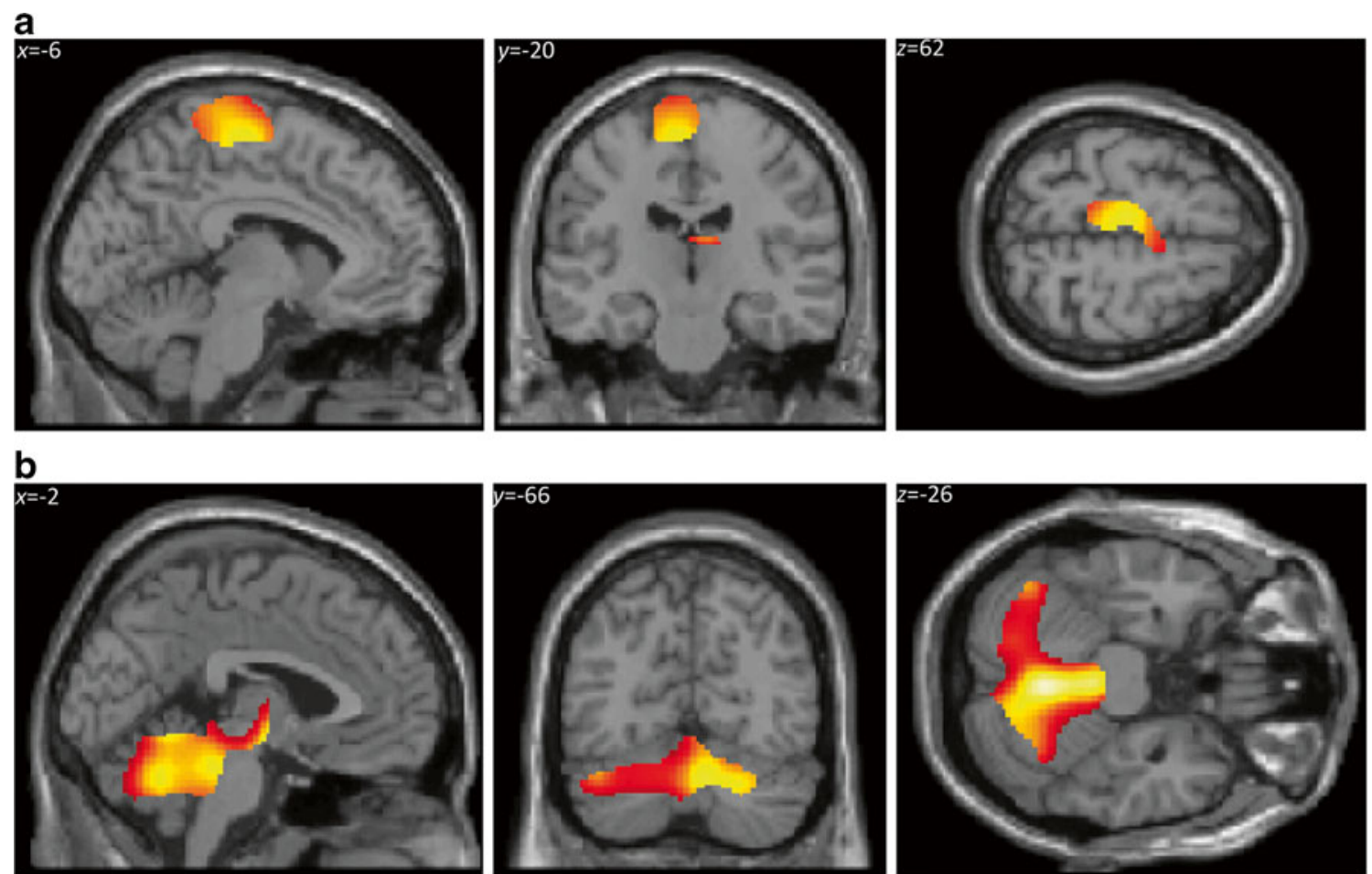

Fig. 2 Statistical parametric maps showing differences in regional cerebral blood flow between organic (DYT1 gene mutation positive) and psychogenic (fixed) dystonia groups, averaged across all three tasks (resting, maintaining a posture, and moving the right lower limb, which was the affected body part in the patients). The statistical parametric maps show regions with relatively increased regional cerebral blood flow $(p<0.05$, corrected for multiple independent comparisons) in either organic dystonia

(a) or psychogenic dystonia (b) within the core motor network. Notably, organic dystonia showed predominantly enhanced cortical regional cerebral blood flow, whereas psychogenic dystonia showed predominantly enhanced subcortical regional cerebral blood flow when these groups were compared with each other. (Reproduced with permission of Oxford University Press from Schrag et al. [60•])

Fig. 3 Statistical parametric maps showing abnormally increased regional cerebral blood flow in dorsolateral/polar prefrontal cortex in both organic dystonia (a) and psychogenic dystonia (b) versus control subjects during movement of the right foot compared with rest (illustrated $p<0.001$, uncorrected). The differential activation in this region was significant $(p<0.05)$ when familywise-corrected within an a priori region of interest defined by Brodmann areas 10 and 46 bilaterally. (Reproduced with permission of Oxford University Press from Schrag et al. [60•])
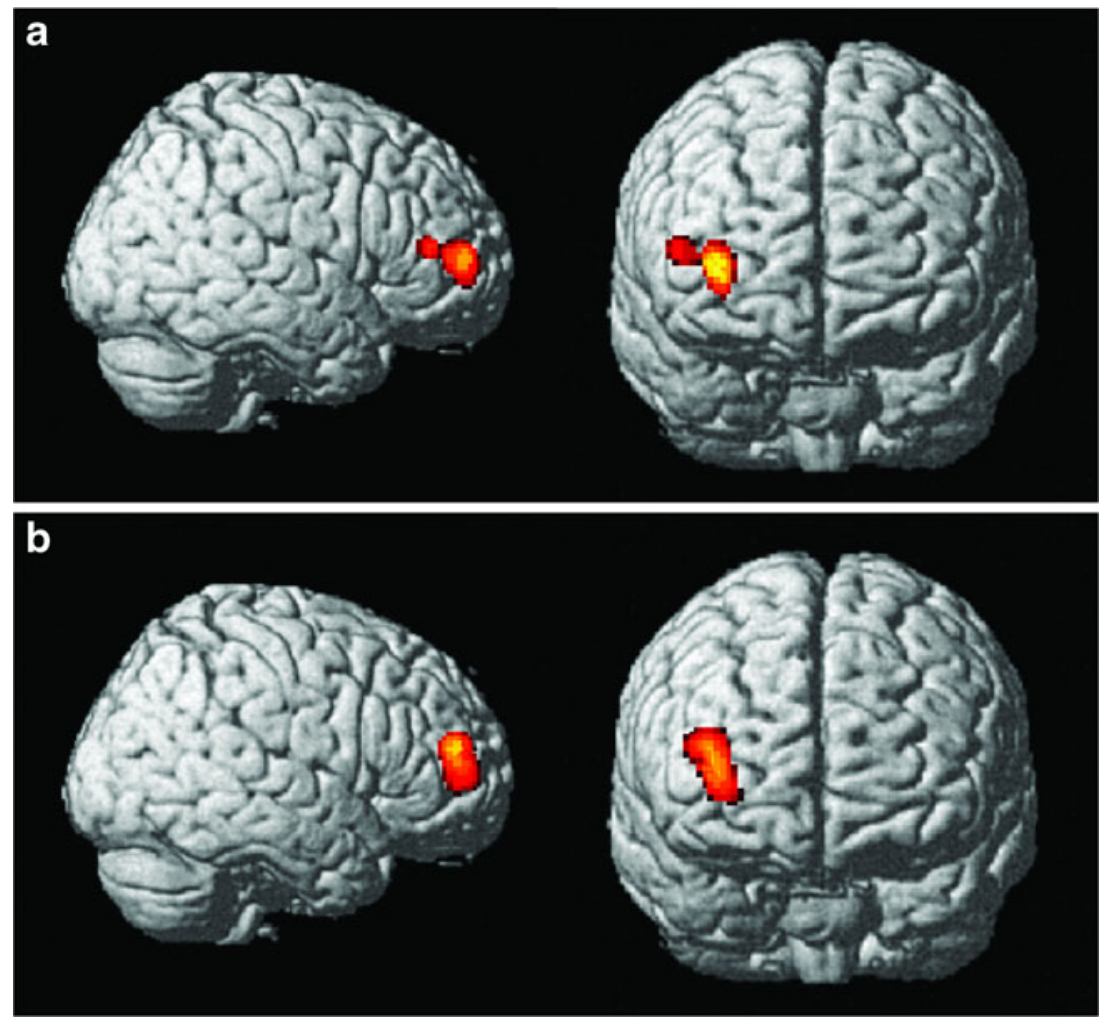
fixed dystonia (Fig. 1) $[12,82]$ and genetically determined (DYT1 gene mutation positive) dystonia, maximizing clinical compatibility within and between groups. It was hypothesized that there would be differences in abnormal brain activation patterns between the two patient groups. In addition, the hypothesis that activation of the prefrontal cortex is a hallmark of a psychogenic cause was challenged. Patients with psychogenic or organic dystonia of the right leg and matched healthy volunteers underwent $\mathrm{H}_{2}{ }^{15} \mathrm{O}$ PET of regional cerebral flow during three tasks in which participants rested, held a fixed posture or undertook paced ankle movements of their right (affected) leg against a footplate dynamometer. Continuous monitoring of the surface electromyogram and footplate manometry were used to monitor task performance. We found that there are anatomically distinct patterns of abnormal regional cerebral blood flow in psychogenic versus organic dystonia. To identify task-independent effects, all three tasks were averaged: patients with organic dystonia were found to have a predominantly cortical pattern of activation, with increased regional cerebral blood flow in primary motor, premotor and parietal cortices, together with reduced flow in subcortical structures, including the cerebellum. In contrast, patients with psychogenic dystonia had an opposite pattern of activation, with abnormal increases in regional cerebral blood flow in subcortical structures, including the striatum and cerebellum, and concomitant reduced perfusion in the primary motor cortex. Not only did the two groups of patients differ from healthy volunteers, they also differed significantly from each other in these regions (Fig. 2). These patterns were present across different motor tasks and at rest, and it is interesting to reflect that these effects of diagnostic group (psychogenic, organic, control) would probably have been missed by fMRI, which relies on task-by-group interactions. The identification of distinct cortical-subcortical neuroimaging features that differentiate organic from psychogenic dystonia complements transcranial magnetic stimulation studies on plasticity in dystonia [83]. Examining the task-dependent effects in the prefrontal cortex, we also identified significant increases in movement-related activation in the right dorsolateral/polar prefrontal cortex in both psychogenic and,

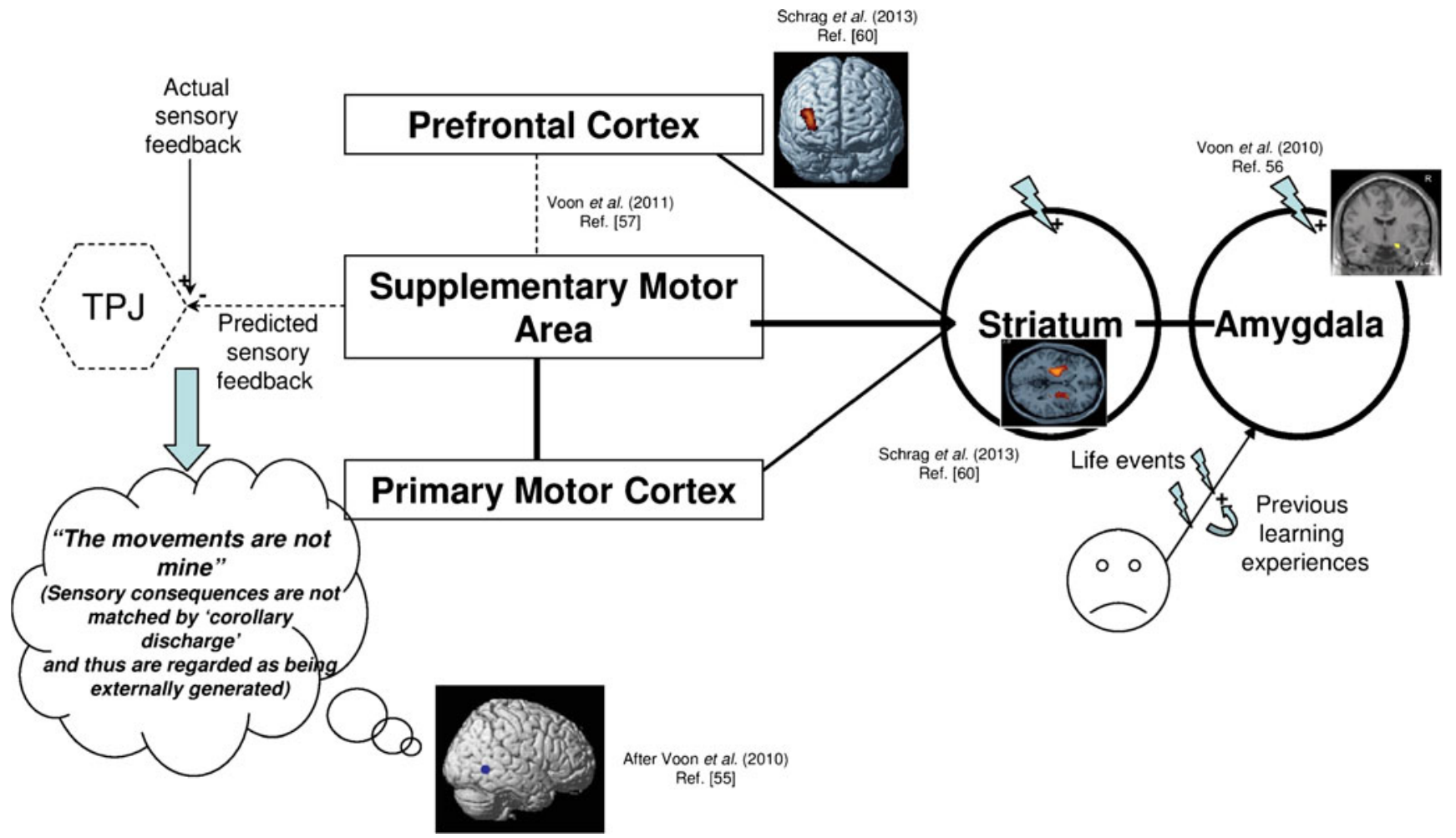

Fig. 4 Possible neural networks involved in psychogenic movement disorders based on the latest advances from the neuroimaging literature. There is an overly sensitive emotional network, possibly conditioned by previous learning experiences, that feeds into the extended motor network via the striatum. In the presence of abnormal self-directed attention, mediated by abnormal prefrontal cortical activation that is functionally disconnected from the core motor network, these changes drive the production of aberrant movements that are not yoked to a normal sense of self-agency. This is because of hypoactivity of the supplementary motor area that normally provides the 'corollary discharge' signal that informs the temporoparietal junction $(T P J)$ 'comparator' what to expect in terms of sensory feedback as a result of internally generated, as opposed to externally generated, movements. As a consequence of the abnormal network activity, the movements are interpreted by patients as being involuntary. An interrupted line denotes a weakened network. (Reproduced with permission of Oxford University Press from Schrag et al. [60•] and Voon et al. [56•]) 
surprisingly, organic dystonia (Fig. 3). This argues against the longstanding proposal that the abnormalities in the prefrontal cortex are a defining feature of psychogenic disorders. This region of prefrontal cortex forms part of an extended motor network (reviewed in [84]) and may be a common substrate in both disorders resulting from changes in motor attention. This supports the aforementioned work suggesting abnormal attentional and emotional control in neural network activity between the amygdala and the supplementary motor area. Although there are no direct projections between these two brain areas, the amygdala projects to the subcortical striatum, which has projections via the thalamus to the supplementary motor area [85]. A subcortical signature specific to psychogenic dystonia might thereby cause abnormal activation of the supplementary motor area via the striatum.

Figure 4 illustrates an emerging schematic overview of possible mechanisms derived from the principal findings from these studies on PMDs to date. In support of this hypothetical framework, recent work by Edwards et al. [61•] provides a holistic neurobiological account, applying a Bayesian and Helmholtzian statistical framework that offers a novel approach to elucidating PMDs. This accommodates several fundamental clinical observations of PMDs and links three different levels of explanation: (1) neuronal synaptic mechanisms; (2) attentional and intentional psychological processes; and (3) computational mechanisms in the brain. In their formulation, PMDs can be understood in terms of pathologically precise beliefs (prior beliefs) at an intermediate level of the motor hierarchy (such as the supplementary motor area) that are focused and released by higher-level attentional processes. The latter processes are modulated by symptom expectations, physical and emotional experiences, and beliefs about illness, resulting in aberrant motor responses by classic lower-level reflex arcs. The consequences of the motor response are not predicted by the dissociated higher-level networks. This model suggests that there is "a symptomatic [mis]interpretation or misattribution of agency to external causes; in short, a failure to realize that the movement was intended". The result is that movements are experienced by patients as being involuntary.

\section{Conclusions}

Neuroimaging of PMDs is beginning to answer the major questions concerning the neurobiology of this group of severe and chronic illnesses. However, there remain many unanswered questions. For instance, what determines the nature of the neurological disorder-tremor, dystonia, myoclonus, pure gait disturbance, parkinsonism, chorea, and tics? What are the differences in the mechanisms underlying each of these clinical entities? Can the imaging findings be developed to help support diagnosis of individual patients in clinical practice? This is more pressing for some clinical scenarios than for others, e.g. patients with possible psychogenic dystonia, which can be difficult to distinguish clinically from organic dystonia. Can we develop novel treatment approaches on the basis of neuroimaging data? For example, if regional changes in neural activation or network dynamics are causal for PMDs, can this be favourably modulated by therapeutic transcranial magnetic stimulation [86] or transcranial direct current stimulation? We propose that an integrated approach to PMDs is required to accelerate the mechanistic understanding and find new treatments. Neuroimaging will continue to play a leading role in such a strategy, for at least two reasons. Pragmatically, fMRI, PET and related methods provide diagnostic stratification of patients and objective outcome markers. Perhaps of even greater importance is the contribution of neuroimaging to answer questions regarding the mechanism and cause.

Acknowledgments Arpan R. Mehta is supported by a National Institute for Health Research Medical Academic Clinical Fellowship. James B. Rowe. is supported by the Wellcome Trust (088324), the National Institute for Health Research Cambridge Biomedical Research Centre and the James S. McDonnell Foundation. The views expressed are those of the authors and not necessarily those of the NHS, the Department of Health or any funding bodies.

\section{Compliance with Ethics Guidelines}

Conflict of Interest Arpan R. Mehta and Anette E. Schrag declare that they have no conflict of interest.

James B. Rowe has received a consulting fee or honorarium from the Medical Research Council and the Wellcome Trust, and has received support for travel to meetings for the study or otherwise as part of the grants from the Wellcome Trust, the Medical Research Council and the James S. McDonnell Foundation. He receives honoraria for teaching from the University of Copenhagen; and he has received a Treaty of Windsor award from the British Council.

Human and Animal Rights and Informed Consent This article does not contain any studies with human or animal subjects performed by any of the authors.

Open Access This article is distributed under the terms of the Creative Commons Attribution License which permits any use, distribution, and reproduction in any medium, provided the original author(s) and the source are credited.

\section{References}

Papers of particular interest, published recently, have been highlighted as:

- Of importance

1. Edwards MJ, Bhatia KP. Functional (psychogenic) movement disorders: merging mind and brain. Lancet Neurol. 2012;11(3):250-260. doi:10.1016/S1474-4422(11)70310-6.

2. Nowak DA, Fink GR. Psychogenic movement disorders: aetiology, phenomenology, neuroanatomical correlates and therapeutic approaches. 
Neuroimage. 2009;47(3):1015-1025. doi:10.1016/j.neuroimage.2009. 04.082 .

3. Ellenstein A, Kranick SM, Hallett M. An update on psychogenic movement disorders. Curr Neurol Neurosci Rep. 2011;11(4):396-403. doi:10.1007/s11910-011-0205-z.

4. Lang AE, Voon V. Psychogenic movement disorders: past developments, current status, and future directions. Mov Disord. 2011;26(6): 1175-1186. doi:10.1002/mds.23571.

5. Batshaw ML, Wachtel RC, Deckel AW, et al. Munchausen's syndrome simulating torsion dystonia. N Engl J Med. 1985;312(22): 1437-1439. doi:10.1056/NEJM198505303122207.

6. Hallett M. Psychogenic movement disorders: a crisis for neurology. Curr Neurol Neurosci Rep. 2006;6(4):269-271.

7. Williams DT, Ford B, Fahn S. Phenomenology and psychopathology related to psychogenic movement disorders. Adv Neurol. 1995;65: 231-257.

8. Factor SA, Podskalny GD, Molho ES. Psychogenic movement disorders: Frequency, clinical profile, and characteristics. J Neurol Neurosurg Psychiatry. 1995;59(4):406-412.

9. Stone J, Carson A, Duncan R, et al. Who is referred to neurology clinics?-the diagnoses made in 3781 new patients. Clin Neurol Neurosurg. 2010;112(9):747-751. doi:10.1016/j.clineuro.2010.05. 011.

10. Kranick SM, Gorrindo T, Hallett M. Psychogenic movement disorders and motor conversion: a roadmap for collaboration between neurology and psychiatry. Psychosomatics. 2011;52(2):109-116. doi:10.1016/j.psym.2010.12.017.

11. Morgante F, Edwards MJ, Espay AJ, et al. Diagnostic agreement in patients with psychogenic movement disorders. Mov Disord. 2012;27(4):548-552. doi:10.1002/mds.24903.

12. Schrag A, Trimble M, Quinn N, et al. The syndrome of fixed dystonia: an evaluation of 103 patients. Brain. 2004;127(10):2360 2372. doi:10.1093/brain/awh262.

13. van Rijn MA, Munts AG, Marinus J, et al. Intrathecal baclofen for dystonia of complex regional pain syndrome. Pain 2009;143(1-2): 41-47. doi:10.1016/j.pain.2009.01.014.

14. Shamy MC. The treatment of psychogenic movement disorders with suggestion is ethically justified. Mov Disord. 2010;25(3):260-264. doi:10.1002/mds.22911.

15. Edwards MJ, Alonso-Canovas A, Schrag A, et al. Limb amputations in fixed dystonia: a form of body integrity identity disorder? Mov Disord. 2011;26(8):1410-1414. doi:10.1002/mds.23671.

16. Stone J, Sharpe M, Rothwell PM, et al. The 12 year prognosis of unilateral functional weakness and sensory disturbance. J Neurol Neurosurg Psychiatry. 2003;74(5):591-596.

17. Jankovic J, Vuong KD, Thomas M. Psychogenic tremor: long-term outcome. CNS Spectr 2006;11(7):501-508.

18. Thomas M, Vuong KD, Jankovic J. Long-term prognosis of patients with psychogenic movement disorders. Parkinsonism Relat Disord. 2006;12(6):382-387. doi:10.1016/j.parkreldis.2006.03.005.

19. Carson A, Stone J, Hibberd C, et al. Disability, distress and unemployment in neurology outpatients with symptoms 'unexplained by organic disease'. J Neurol Neurosurg Psychiatry. 2011;82(7):810813. doi:10.1136/jnnp.2010.220640.

20. Kanaan RA, Armstrong D, Wessely SC. Neurologists' understanding and management of conversion disorder. J Neurol Neurosurg Psychiatry. 2011;82(9):961-966. doi:10.1136/jnnp.2010.233114.

21. Espay AJ, Goldenhar LM, Voon V, et al. Opinions and clinical practices related to diagnosing and managing patients with psychogenic movement disorders: An international survey of movement disorder society members. Mov Disord 2009;24(9):1366-1374. doi: 10.1002/mds. 22618 .

22. Browning M, Fletcher P, Sharpe M. Can neuroimaging help us to understand and classify somatoform disorders? A systematic and critical review. Psychosom Med. 2011;73(2):173-184. doi:10.1097/ PSY.0b013e31820824f6.
23. Atmaca M, Aydin A, Tezcan E, et al. Volumetric investigation of brain regions in patients with conversion disorder. Prog Neuropsychopharmacol Biol Psychiatry. 2006;30(4):708-713. doi:10.1016/j.pnpbp.2006.01.011.

24. Kagi G, Bhatia KP, Tolosa E. The role of DAT-SPECT in movement disorders. J Neurol Neurosurg Psychiatry 2010;81(1):5-12. doi:10. 1136/jnnp.2008.157370.

25. Felicio AC, Shih MC, Godeiro-Junior C, et al. Molecular imaging studies in Parkinson disease: reducing diagnostic uncertainty. Neurologist. 2009;15(1):6-16. doi:10.1097/NRL.0b013e318183fdd8.

26. Marek K, Jennings D, Seibyl J. Imaging the dopamine system to assess disease-modifying drugs: studies comparing dopamine agonists and levodopa. Neurology. 2003;61(6 Suppl 3):S 43-48.

27. Parkinson Study Group. A randomized controlled trial comparing pramipexole with levodopa in early Parkinson's disease: design and methods of the CALM-PD study. Clin Neuropharmacol. 2000;23(1):34-44.

28. Whone AL, Watts RL, Stoessl AJ, et al. Slower progression of Parkinson's disease with ropinirole versus levodopa: the REALPET study. Ann Neurol. 2003;54(1):93-101. doi:10.1002/ana.10609.

29. Hallett M, Rothwell J. Milestones in clinical neurophysiology. Mov Disord 2011;26(6):958-967. doi:10.1002/mds.23572.

30. Friston KJ, Buechel C, Fink GR, et al. Psychophysiological and modulatory interactions in neuroimaging. Neuroimage. 1997;6(3): 218-229. doi:10.1006/nimg.1997.0291.

31. Roebroeck A, Formisano E, Goebel R. The identification of interacting networks in the brain using fMRI: Model selection, causality and deconvolution. Neuroimage. 2011;58(2):296-302. doi:10.1016/j.neuroimage.2009.09.036.

32. Friston K, Moran R, Seth AK. Analysing connectivity with Granger causality and dynamic causal modelling. Curr Opin Neurobiol, 2013;23(2):172-178. doi:10.1016/j.conb.2012.11.010.

33. Edwards MJ, Moretto G, Schwingenschuh P, et al. Abnormal sense of intention preceding voluntary movement in patients with psychogenic tremor. Neuropsychologia. 2011;49(9):2791-2793. doi:10. 1016/j.neuropsychologia.2011.05.021.

34. Kranick SM, Moore JW, Yusuf N, et al. Action-effect binding is decreased in motor conversion disorder: implications for sense of agency. Mov Disord. 2013;28(8):1110-1116. doi:10.1002/mds.25408.

35. Parees I, Saifee TA, Kassavetis P, et al. Believing is perceiving: mismatch between self-report and actigraphy in psychogenic tremor. Brain. 2012;135(1):117-123. doi:10.1093/brain/awr292.

36. Parees I, Kassavetis P, Saifee TA, et al. 'Jumping to conclusions' bias in functional movement disorders. J Neurol Neurosurg Psychiatry. 2012;83(4):460-463. doi:10.1136/jnnp-2011-300982.

37. Morgante F, Tinazzi M, Squintani G, et al. Abnormal tactile temporal discrimination in psychogenic dystonia. Neurology. 2011;77(12): 1191-1197. doi:10.1212/WNL.0b013e31822f0449.

38. Mehta AR, Rowe JB, Trimble MR, et al. Coactivation sign in fixed dystonia. Parkinsonism Relat Disord. 2013;19(4):474-476. doi:10. 1016/j.parkreldis.2012.10.014.

39. Schwingenschuh P, Katschnig P, Seiler S, et al. Moving toward "laboratory-supported" criteria for psychogenic tremor. Mov Disord. 2011;26(14);2509-2515. doi:10.1002/mds.23922.

40. Deuschl G, Köster B, Lücking CH, et al. Diagnostic and pathophysiological aspects of psychogenic tremors. Mov Disord. 1998;13(2): 294-302. doi:10.1002/mds.870130216.

41. Tiihonen J, Kuikka J, Viinamaki H, et al. Altered cerebral blood flow during hysterical paresthesia. Biol Psychiatry. 1995;37(2):134-135. doi:10.1016/0006-3223(94)00230-Z.

42. Marshall JC, Halligan PW, Fink GR, et al. The functional anatomy of a hysterical paralysis. Cognition. 1997;64(1):B1-8.

43. Yazici KM, Kostakoglu L. Cerebral blood flow changes in patients with conversion disorder. Psychiatry Res. 1998;83(3):163-168.

44. Spence SA, Crimlisk HL, Cope H, et al. Discrete neurophysiological correlates in prefrontal cortex during hysterical and feigned disorder 
of movement. Lancet 2000;355(9211):1243-1244. doi:10.1016/ S0140-6736(00)02096-1.

45. Vuilleumier P, Chicherio C, Assal F, et al. Functional neuroanatomical correlates of hysterical sensorimotor loss. Brain. 2001;124(6): 1077-1090.

46. Mailis-Gagnon A, Giannoylis I, Downar J, et al. Altered central somatosensory processing in chronic pain patients with "hysterical" anesthesia. Neurology. 2003;60(9):1501-1507.

47. Werring DJ, Weston L, Bullmore ET, et al. Functional magnetic resonance imaging of the cerebral response to visual stimulation in medically unexplained visual loss. Psychol Med. 2004;34(4):583589. doi:10.1017/S0033291703008985.

48. Burgmer M, Konrad C, Jansen A, et al. Abnormal brain activation during movement observation in patients with conversion paralysis. Neuroimage. 2006;29(4):1336-1343. doi:10.1016/j.neuroimage. 2005.08.033.

49. Ghaffar O, Staines WR, Feinstein A. Unexplained neurologic symptoms: an fMRI study of sensory conversion disorder. Neurology. 2006;67(11):2036-2038. doi:10.1212/01.wnl.0000247275.68402.fc.

50. de Lange FP, Roelofs K, Toni I. Increased self-monitoring during imagined movements in conversion paralysis. Neuropsychologia. 2007;45(9):2051-2058. doi:10.1016/j.neuropsychologia.2007.02.002.

51. Kanaan RA, Craig TK, Wessely SC, et al. Imaging repressed memories in motor conversion disorder. Psychosom Med. 2007;69(2): 202-205. doi:10.1097/PSY.0b013e31802e4297.

52. Stone J, Zeman A, Simonotto E, et al. FMRI in patients with motor conversion symptoms and controls with simulated weakness. Psychosom Med. 2007;69(9):961-969. doi:10.1097/PSY.0b013e31815b6c14.

53. Cojan Y, Waber L, Carruzzo A, et al. Motor inhibition in hysterical conversion paralysis. Neuroimage. 2009;47(3):1026-1037. doi:10. 1016/j.neuroimage.2009.05.023.

54. de Lange FP, Toni I, Roelofs K. Altered connectivity between prefrontal and sensorimotor cortex in conversion paralysis. Neuropsychologia. 2010;48(6):1782-1788. doi:10.1016/j.neuropsychologia.2010.02.029.

55. - Voon V, Gallea C, Hattori N, et al. The involuntary nature of conversion disorder. Neurology. 2010;74(3):223-228. doi:10.1212/ WNL.0b013e3181ca00e9. Psychogenic tremor was associated with right temporoparietal junction hypoactivity and lower interactions between this area and the sensorimotor cortex. The right temporoparietal junction is thought to be an important comparator region, comparing actual with predicted sensory feedback. The absence of a feedforward signal would lead to a mismatch in the temporoparietal junction, such that the actual feedback would be received 'unchecked', owing to a lack of predicted sensory feedback (corollary discharge). This is hypothesized to provoke the feeling of involuntariness associated with movement: a lack of agency.

56. - Voon V, Brezing C, Gallea C, et al. Emotional stimuli and motor conversion disorder. Brain. 2010;133(5):1526-1536. doi:10.1093/ brain/awq054. This study demonstrated abnormally strong amygdala-supplementary motor area connectivity when patients with PMDs were presented with emotionally valent stimuli. The heightened amygdala activity to arousal is hypothesized to have downstream effects on motor initiation and nonconscious response inhibition.

57. - Voon V, Brezing C, Gallea C, et al. Aberrant supplementary motor complex and limbic activity during motor preparation in motor conversion disorder. Mov Disord. 2011;26(13):2396-2403. doi:10.1002/ mds.23890. This study showed reduced activity in the supplementary motor area in PMDs and increased activity in the amygdala and other areas implicated in emotional salience. It also showed reduced functional connectivity between the supplementary motor area and bilateral prefrontal cortices for internally generated movements. This is hypothesized to account for a potential impairment in top-down regulation of motor control to guide action selection.

58. Czarnecki K, Jones DT, Burnett MS, et al. SPECT perfusion patterns distinguish psychogenic from essential tremor. Parkinsonism Relat Disord. 2011;17(5):328-332. doi:10.1016/j.parkreldis.2011.01.012.
59. Hedera P. Metabolic hyperactivity of the medial posterior parietal lobes in psychogenic tremor. Tremor Other Hyperkinet Mov (N Y) 2012;2:tre-02-50-441-1.

60. - Schrag AE, Mehta AR, Bhatia KP, et al. The functional neuroimaging correlates of psychogenic versus organic dystonia. Brain. 2013;136(3): 770-781. doi:10.1093/brain/awt008. This article demonstrated a subcortical-cortical differentiation between organic (DYT1 gene mutation positive) and psychogenic (fixed) dystonia in terms of regional cerebral perfusion, with the latter patient group being characterized by abnormally increased perfusion in the cerebellum and basal ganglia, and abnormally decreased perfusion in the primary motor cortex (medial leg area); the opposite pattern was demonstrated in organic dystonia. The celebrated classic finding of abnormal prefrontal cortical activation in psychogenic neurological disorders was shown to not be exclusive to the psychogenic cause, because it was also seen in the organic dystonia group. The authors suggest that derangements in the mechanisms of motor attention may be a feature common to both conditions.

61. - Edwards MJ, Adams RA, Brown H, et al. A Bayesian account of 'hysteria'. Brain. 2012;135(11):3495-3512. doi:10.1093/brain/aws129. This article provides a cogent account invoking a Bayesian hierarchical model to explain functional motor and sensory symptoms - they are pathologically precise prior beliefs modulated by attentional processes, resulting in perceptual and/or motor symptoms (posterior beliefs) that are experienced by the patient as being involuntary.

62. Rowe JB. Conversion disorder: understanding the pathogenic links between emotion and motor systems in the brain. Brain. 2010;133(5): 1295-1297. doi:10.1093/brain/awq096.

63. Halligan PW, Athwal BS, Oakley DA, et al. Imaging hypnotic paralysis: implications for conversion hysteria. Lancet. 2000;355(9208):986-987. doi:10.1016/S0140-6736(00)99019-6.

64. Ward NS, Oakley DA, Frackowiak RS, et al. Differential brain activations during intentionally simulated and subjectively experienced paralysis. Cogn Neuropsychiatry. 2003;8(4):295-312. doi:10. 1080/13546800344000200.

65. Roelofs K, van Galen GP, Keijsers GP, et al. Motor initiation and execution in patients with conversion paralysis. Acta Psychol (Amst). 2002;110(1):21-34

66. Roelofs K, de Bruijn ERA, Van Galen GP. Hyperactive action monitoring during motor-initiation in conversion paralysis: an eventrelated potential study. Biol Psychol 2006;71(3):316-325. doi:10. 1016/j.biopsycho.2005.07.002.

67. de Lange FP, Roelofs K, Toni I. Motor imagery: a window into the mechanisms and alterations of the motor system. Cortex. 2008;44(5): 494-506. doi:10.1016/j.cortex.2007.09.002.

68. Vuilleumier P. The neurophysiology of self-awareness disorders in conversion hysteria. In: Laureys S, Tononi G, editors. The neurology of consciousness: cognitive neuroscience and neuropathology. Amsterdam: Academic; 2009. p. 282-302.

69. Burgess PW, Dumontheil I, Gilbert SJ. The gateway hypothesis of rostral prefrontal cortex (area 10) function. Trends Cogn Sci. 2007;11(7):290-298. doi:10.1016/j.tics.2007.05.004.

70. Bhatia KP, Schneider SA. Psychogenic tremor and related disorders. J Neurol. 2007;254(5):569-574. doi:10.1007/s00415-006-0348-z.

71. Hallett M, Fahn S, Jankovic J, et al., editors. Psychogenic movement disorders: neurology and neuropsychiatry. Philadelphia: Lippincott Williams \& Wilkins; 2005.

72. Krem MM. Motor conversion disorders reviewed from a neuropsychiatric perspective. J Clin Psychiatry. 2004;65(6):783-790.

73. Bogousslavsky J. Hysteria after Charcot: back to the future. Front Neurol Neurosci. 2011;29:137-161. doi:10.1159/000321783.

74. Seignourel PJ, Miller K, Kellison I, et al. Abnormal affective startle modulation in individuals with psychogenical movement disorder. Mov Disord. 2007;22(9):1265-1271. doi:10.1002/mds.21451.

75. Horvath T, Friedman J, Meares R. Attention in hysteria: a study of Janet's hypothesis by means of habituation and arousal measures. Am J Psychiatry. 1980;137(2):217-220. 
76. Lader M, Sartorius N. Anxiety in patients with hysterical conversion symptoms. J Neurol Neurosurg Psychiatry. 1968;31(5):490-495.

77. Hallett M. Physiology of psychogenic movement disorders. J Clin Neurosci. 2010;17(8):959-965. doi:10.1016/j.jocn.2009.11.021.

78. Shibasaki H, Hallett M. What is the Bereitschaftspotential? Clin Neurophysiol. 2006;117(11):2341-2356. doi:10.1016/j.clinph.2006. 04.025.

79. Farrer C, Frith CD. Experiencing oneself vs another person as being the cause of an action: the neural correlates of the experience of agency. Neuroimage. 2002;15(3):591-603. doi:10.1006/nimg. 2001.1009.

80. Blakemore SJ, Sirigu A. Action prediction in the cerebellum and in the parietal lobe. Exp Brain Res. 2003;153(2):239-245. doi:10.1007/s00221003-1597-z.

81. Schnell K, Heekeren K, Daumann J, et al. Correlation of passivity symptoms and dysfunctional visuomotor action monitoring in psychosis. Brain. 2008;131(10):2783-2797. doi:10.1093/brain/awn184.
82. Ibrahim NM, Martino D, van de Warrenburg BP, et al. The prognosis of fixed dystonia: a follow-up study. Parkinsonism Relat Disord. 2009;15(8):592-597. doi:10.1016/j.parkreldis.2009. 02.010 .

83. Quartarone A, Rizzo V, Terranova C, et al. Abnormal sensorimotor plasticity in organic but not in psychogenic dystonia. Brain. 2009;132(10):2871-2877. doi:10.1093/brain/awp213.

84. Rowe JB, Siebner HR. The motor system and its disorders. Neuroimage. 2012;61(2):464-477. doi:10.1016/j.neuroimage.2011. 12.042 .

85. Groenewegen HJ, Wright CI, Uylings HB. The anatomical relationships of the prefrontal cortex with limbic structures and the basal ganglia. J Psychopharmacol. 1997;11(2):99-106.

86. Pollak TA, Nicholson TR, Edwards MJ, et al. A systematic review of transcranial magnetic stimulation in the treatment of functional (conversion) neurological symptoms. J Neurol Neurosurg Psychiatry. 2013. doi:10.1136/jnnp-2012-304181. 